\title{
Mechanism for the Intermittent Route to Strange Nonchaotic Attractors
}

\author{
Sang-Yoon Kim,, , , 用 Woochang Lim, ${ }^{2}$ and Edward Ott ${ }^{1}$ 用 \\ ${ }^{1}$ Institute for Research in Electronics and Applied Physics, \\ University of Maryland, College Park, Maryland 20742 \\ ${ }^{2}$ Department of Physics, Kangwon National University, Chunchon, Kangwon-Do 200-701, Korea
}

\begin{abstract}
Intermittent strange nonchaotic attractors (SNAs) appear typically in quasiperiodically forced period-doubling systems. As a representative model, we consider the quasiperiodically forced logistic map and investigate the mechanism for the intermittent route to SNAs using rational approximations to the quasiperiodic forcing. It is thus found that a smooth torus is transformed into an intermittent SNA via a phase-dependent saddle-node bifurcation when it collides with a new type of "ring-shaped" unstable set. Besides this intermittent transition, other transitions such as the interior, boundary, and band-merging crises may also occur through collision with the ring-shaped unstable sets. Hence the ring-shaped unstable sets play a central role for such dynamical transitions. Furthermore, these kinds of dynamical transitions seem to be "universal," in the sense that they occur typically in a large class of quasiperiodically forced period-doubling systems.
\end{abstract}

PACS numbers: 05.45.Ac, 05.45.Df, 05.45.Pq

Recently much attention has been paid to the study of quasiperiodically forced systems because of the generic appearance of strange nonchaotic attractors (SNAs) [1]. SNAs were first described by Grebogi et al. [2] and since then have been extensively investigated both numerically 近, 田, 6, 6, 1, 8, 9, 10, 11, 12, 13, 14, 15, 16, and experimentally [17]. SNAs exhibit some properties of regular as well as chaoic attractors. Like regular attractors, their dynamics is nonchaotic; like typical chaotic attractors, they exhibit fractal phase space structure. Furthermore, SNAs are related to Anderson localization in the Schrödinger equation with a spatially quasiperiodic potential [18], and they may have a practical application in secure communication [19]. Therefore, dynamical transitions in quasiperiodically forced systems have become a topic of considerable current interest. However, their mechanisms are still much less understood than those in unforced or periodically forced systems.

Here we are interested in the dynamical transition to SNAs accompanied by intermittent behavior, as reported in [12]. As a parameter passes a threshold value, a smooth torus is abruptly transformed into an intermittent SNA. Near the transition point, the intermittent dynamics on the SNA was characterized in terms of the average interburst time and the Lyapunov exponent. This route to an intermittent SNA is quite general and has been observed in a number of quasiperiodically forced period-doubling maps and flows (e.g., see [13, 14]). It has been suggested [15] that the observed intermittent behavior results through interaction with an unstable orbit. However, in the previous work, such an unstable orbit was not located, and thus the bifurcation mechanism for the intermittent transition still remains unclear.

In this paper, we study the underlying mechanism of the intermittency in the quasiperiodically forced logistic map $M$ [0] which is a representative model for the quasiperiodically forced period-doubling systems:

$$
M:\left\{\begin{array}{l}
x_{n+1}=\left(a+\varepsilon \cos 2 \pi \theta_{n}\right) x_{n}\left(1-x_{n}\right), \\
\theta_{n+1}=\theta_{n}+\omega(\bmod 1),
\end{array}\right.
$$

where $x \in[0,1], \theta \in S^{1}, a$ is the nonlinearity parameter of the logistic map, and $\omega$ and $\varepsilon$ represent the frequency and amplitude of the quasiperiodic forcing, respectively. We set the frequency to be the reciprocal of the golden mean, $\omega=(\sqrt{5}-1) / 2$. The intermittent transition is then investigated using the rational approximations (RAs) to the quasiperiodic forcing. For the case of the inverse golden mean, its rational approximants are given by the ratios of the Fibonacci numbers, $\omega_{k}=F_{k-1} / F_{k}$, where the sequence of $\left\{F_{k}\right\}$ satisfies $F_{k+1}=F_{k}+F_{k-1}$ with $F_{0}=0$ and $F_{1}=1$. Instead of the quasiperiodically forced system, we study an infinite sequence of periodically forced systems with rational driving frequencies $\omega_{k}$. We suppose that the properties of the original system $M$ may be obtained by taking the quasiperiodic limit $k \rightarrow \infty$. Using this technique we observe a new type of invariant unstable set, which will be referred to as the "ring-shaped" unstable set in accordance with its geometry. When a smooth torus (corresponding to an ordinary quasiperiodic attractor) collides with this ringshaped unstable set, a transition to an intermittent SNA is found to occur.

We also note that the quasiperiodically forced logistic map $M$ is noninvertible, because its Jacobian determinant becomes zero along the critical curve, $L_{0}=$ $\{x=0.5, \theta \in[0,1)\}$. Critical curves of rank $k, L_{k}$ $(k=1,2, \ldots)$, are then given by the images of $L_{0}$, [i.e., $\left.L_{k}=M^{k}\left(L_{0}\right)\right]$. Segments of these critical curves can be used to define a bounded trapping region of the phase space, called an "absorbing area," inside which, upon entering, trajectories are henceforth confined [20]. It is found that the newly-born intermittent SNA fills the absorbing area. Hence the global structure of the SNA is 

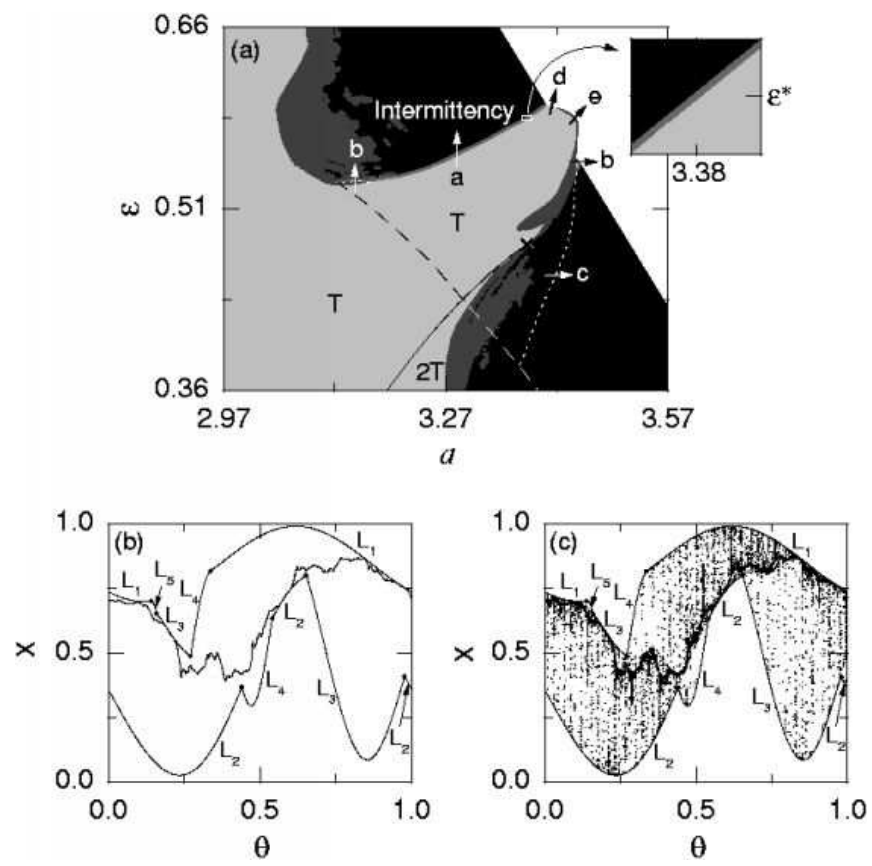

FIG. 1: (a) Phase Diagram in the $a-\varepsilon$ plane. Regular, chaotic, SNA, and divergence regimes are shown in light gray, black, gray (or dark gray), and white, respectively. To show the region of existence (gray) of the intermittent SNA occurring between $T$ (light gray) and the chaotic attractor region (black), a small box near $(a, \varepsilon)=\left[3.38, \varepsilon^{*}(=0.584726781)\right]$ is magnified. Through interaction with the ring-shaped unstable set born when passing the dashed line, typical dynamical transitions such as the intermittency (route $a$ ) and the interior (routes $b$ and $c$; the dotted line is an interior crisis line) and boundary (routes $d$ and $e$ ) crises may occur. Here the torus and the doubled torus are denoted by $T$ and $2 T$, and the solid line represents a torus doubling bifurcation curve. (b) Smooth torus inside an absorbing area with boundary formed by segments of the critical curves $L_{k}(k=1, \ldots, 5)$ for $a=3.38$ and $\varepsilon=0.5847$. (c) SNA filling the absorbing area for $a=3.38$ and $\varepsilon=0.58475$. For other details, see the text.

determined by the critical curves.

Figure 1(a) shows a phase diagram in the $a-\varepsilon$ plane. Each phase is characterized by the Lyapunov exponent $\sigma_{x}$ in the $x$-direction as well as the phase sensitivity exponent $\delta$. The exponent $\delta$ measures the sensitivity with respect to the phase of the quasiperiodic forcing and was introduced in [6] to characterize the strangeness of an attractor. A smooth torus that has a negative Lyapunov exponent without phase sensitivity $(\delta=0)$ exists in the region denoted by $T$ and shown in light gray. Upon crossing the solid line, the smooth torus becomes unstable and bifurcates to a smooth doubled torus in the region denoted by $2 T$. Chaotic attractors with positive Lyapunov exponents exist in the region shown in black. Between these regular and chaotic regions, SNAs that have negative Lyapunov exponents with high phase sensitivity $(\delta>0)$ exist in the regions shown in gray and dark gray. Consistent with their positive phase sensitivity exponent $\delta$, these SNAs are observed to have fractal structure [6]. Here we restrict our considerations only to the intermittent SNAs that exist in the thin gray region [e.g., see a magnified part in Fig. 1(a)]. (In the darkgray region, nonintermittent SNAs, born through other mechanisms, such as gradual fractalization [10] and torus collision [5], exist.) Note that this phase diagram is typical for quasiperiodically forced period-doubling systems $12,13,14,15,16,21$, and its main interesting feature is the existence of the "tongue" of quasiperiodic motion that penetrates into the chaotic region and separates it into upper and lower parts. We also note that this tongue lies near the terminal point (denoted by the cross) of the torus doubling bifurcation curve. When crossing the upper boundary of the tongue, a smooth torus is transformed into an intermittent SNA that exists in the thin gray region. Hereafter this intermittent route to SNAs will be referred to as the route $a$ [see Fig. 1](a)].

As an example, we consider the case $a=3.38$. Figure $\mathbb{1}(\mathrm{b})$ shows a smooth torus with $\sigma_{x}=-0.059$ for $\varepsilon=0.5847$ inside an absorbing area whose boundary is formed by segments of the critical curves $L_{k}(k=$ $1, \ldots, 5)$ (the dots indicate where these segments connect). We also note that the smooth unstable torus $x=0$ and its first preimage $x=1$ form the basin boundary of the smooth torus in the $\theta-x$ plane. However, as $\varepsilon$ passes a threshold value $\varepsilon^{*}(=0.584726781)$, a transition to an intermittent SNA occurs. As shown in Fig. 1(c) for $\varepsilon=0.58475$, the newly-born intermittent SNA with $\sigma_{x}=-0.012$ and $\delta=19.5$ appears to fill the absorbing area, and its typical trajectory spends most of its time near the former torus with sporadic large bursts away from it. This intermittent transition may be expected to have occurred through collision of the smooth attracting torus with an unstable orbit. However, the smooth unstable torus $x=0$ cannot interact with the smooth stable torus, because it lies outside the absorbing area. Hence we search inside the absorbing area for an unstable orbit that might collide with the smooth stable torus.

Using RAs we find a new type of ring-shaped unstable set that causes the intermittent transition through collision with the smooth torus. When passing the dashed curve in Fig. 1 (a), such a ring-shaped unstable set appears via a phase-dependent saddle-node bifurcation. This bifurcation has no counterpart in the unforced case. (The dashed line is numerically obtained for the sufficiently large level $k=10$ of the RAs.) For each $\mathrm{RA}$ of level $k$, a periodically forced logistic map with the rational driving frequency $\omega_{k}$ has a periodic or chaotic attractor that depends on the initial phase $\theta_{0}$ of the external force. Then the union of all attractors for different $\theta_{0}$ gives the $k$ th approximation to the attractor in the quasiperiodically forced system. As an example, consider the RA of low level $k=6$. As shown in Fig. 2(a) for $a=3.246$ and $\varepsilon=0.446$, the RA to the smooth 


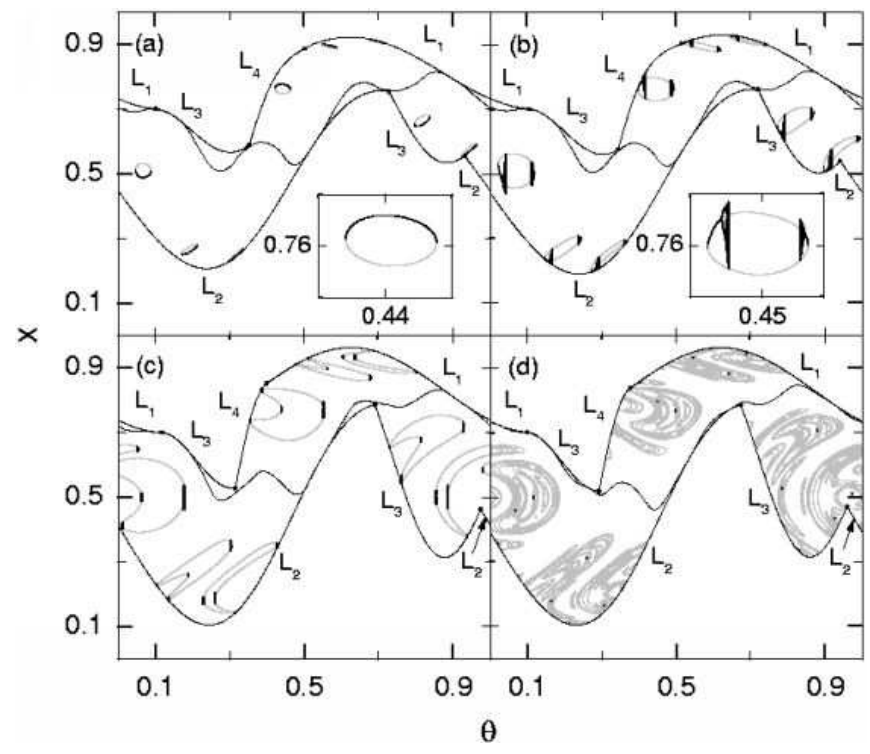

FIG. 2: Ring-shaped unstable sets in the RA of level 6 for (a) $a=3.246$ and $\varepsilon=0.446$, (b) $a=3.26$ and $\varepsilon=0.46$, and (c) $a=3.326$ and $\varepsilon=0.526$. (d) Ring-shaped unstable set in the RA of level 8 for $a=3.326$ and $\varepsilon=0.526$. These ring-shaped unstable sets exist inside the absorbing area with boundary formed by portions of the critical curves $L_{k}(k=1, \ldots, 4)$

torus, consisting of stable orbits with period $F_{6}(=8)$, exists inside an absorbing area bounded by segments of the critical curves $L_{k}(k=1, \ldots, 4)$. Note also that a ring-shaped unstable set, born via a phase-dependent saddle-node bifurcation and composed of 8 small rings, lies inside the absorbing area. At first, each ring consists of the stable (shown in black) and unstable (shown in gray) orbits with the forcing period $F_{6}(=8)$ [see the inset in Fig. 2(a)]. However, as the parameters increase, a chaotic attractor appears through period doublings of the stable periodic orbit, and then it disappears through collision with the unstable $F_{6}$-periodic orbit (see Fig. 2(b) for $a=3.26$ and $\varepsilon=0.46$ ). With further increase in the parameters, both the size and shape of the rings change, and for sufficiently large parameters, each ring consists of a large unstable part (shown in gray) and a small attracting part (shown in black), as shown in Fig. 2(c) for $a=3.326$ and $\varepsilon=0.526$. [Note that the unstable part (plotted in the gray) of each ring consists of only unstable orbits whose period is the same as that of the forcing, i.e., $F_{k}$.] For the same parameter values as in Fig. 2(c), we increase the level of the RA to $k=8$. Then the number of rings $(=336)$ increases significantly, and the unstable part [shown in gray and consisting of unstable orbits with period $F_{8}(=21)$ ] becomes dominant, because the attached attracting part (shown in black) becomes negligibly small [see Fig. 2(d)]. In this way, with increasing the level $k$ the ring-shaped unstable set consists of a larger number of rings with smaller attracting part. Hence, in the quasiperiodic limit these ring-shaped
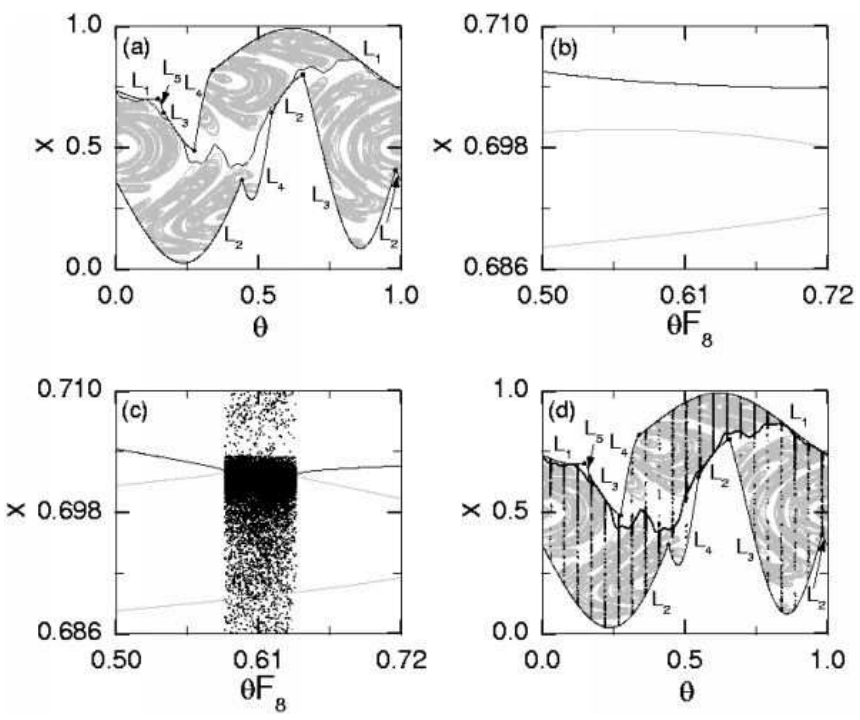

FIG. 3: (a) and (b) RA to the smooth torus (shown in black) and the ring-shaped unstable set (shown in gray) in the RA of level $8\left(F_{8}=21\right)$ for $a=3.38$ and $\varepsilon=0.586$. (c) and (d) 8th RA to the SNA for $a=3.38$ and $\varepsilon=0.5864$. For more details, see the text.

unstable sets seem to form a complicated unstable set composed of only unstable orbits.

In what follows we use RAs to explain the mechanism for the intermittent transition occurring in Figs. 1 1 (b) and 1 (c) for $a=3.38$. Figures 3(a) and 3(b) show that, inside the absorbing area, the ring-shaped unstable set (shown in gray) lies very close to the smooth torus (shown in black) for $\varepsilon=0.586$ in the RA of level $k=8$. As $\varepsilon$ passes a threshold value $\varepsilon_{8}(=0.586366)$, a phasedependent saddle-node bifurcation occurs via a collision between the smooth torus and the ring-shaped unstable set, and then "gaps," where no orbits with period $F_{8}$ $(=21)$ exist, are formed. A magnified gap is shown in Fig. 3(c) for $\varepsilon=0.5846$. Note that this gap is filled by intermittent chaotic attractors together with orbits with period higher than $F_{8}$ embedded in very small windows. As shown in Fig. 3(d), the RA to the whole attractor consists of the union of the periodic component and the intermittent chaotic component, where the latter occupies the 21 gaps in $\theta$ and is vertically bounded by segments of the critical curves $L_{k}(k=1, \ldots, 5)$. However, the periodic component is dominant: the average Lyapunov exponent $\left(\left\langle\sigma_{x}\right\rangle=-0.09\right)$ is negative, where $\langle\cdots\rangle$ denotes the average over the whole $\theta$. We note that Fig. 3(d) seems to be similar to Fig. 1 1(c), although the level $k=8$ is low. Increasing the level up to $k=15$ we find that the threshold value $\varepsilon_{k}$ at which the phase-dependent saddle-node bifurcation occurs converges to the quasiperiodic limit $\varepsilon^{*}(=0.584726781)$ in an algebraic way, $\left|\Delta \varepsilon_{k}\right| \sim F_{k}^{-\alpha}$, where $\Delta \varepsilon_{k}=\varepsilon_{k}-\varepsilon^{*}$ and $\alpha \simeq 2.2$ [21]. Furthermore, in the quasiperiodic limit $k \rightarrow \infty$ the RA to the attractor has a dense set 
of gaps that are filled by intermittent chaotic attractors and bounded by portions of the critical curves. Thus, an intermittent SNA, containing the ring-shaped unstable set and filling the absorbing area, appears, as shown in Fig. 11(c). In addition, we find that as $\varepsilon$ passes another threshold value $\varepsilon_{c}(=0.5848)$, this SNA is transformed into a chaotic attractor with a positive Lyapunov exponent. Using the RA, this transition to chaos may also be explained. For each RA, its angle averaged Lyapunov exponent $\left\langle\sigma_{x}\right\rangle$ is given by the sum of the "weighted" Lyapunov exponents of the periodic and chaotic components of the RA, $\Lambda_{p}$ and $\Lambda_{c}$, (i.e. $<\sigma_{x}>=\Lambda_{p}+\Lambda_{c}$ ), where $\Lambda_{p(c)} \equiv M_{p(c)}<\sigma_{x}>_{p(c)}$, and $M_{p(c)}$ and $<\sigma>_{p(c)}$ are the Lebesgue measure in $\theta$ and average Lyapunov exponent of the periodic (chaotic) component, respectively. After passing a threshold value where the magnitude of $\Lambda_{p}$ and $\Lambda_{c}$ are balanced, the chaotic component becomes dominant, and hence a chaotic attractor appears. (More details will be given in Ref. [21].)

To sum up, using RAs we have found the mechanism for the intermittent route to SNAs in the quasiperiodically forced logistic map. When a smooth torus makes a collision with a new type of ring-shaped unstable set, a transition to an intermittent SNA, bounded by segments of critical curves, occurs via a phase-dependent saddle-node bifurcation. Other typical transitions such as interior [routes b and c in Fig. 11(a)] and boundary [routes d and e in Fig. 11(a)] crises may also occur near the main tongue through interaction with the ring-shaped unstable set [21]. Furthermore, as $\varepsilon$ decreases toward zero, similar tongues appear successively near the terminal points of the higher-order torus-doubling bifurcations [22], and band-merging crises may also occur through collision with the ring-shaped unstable set 21]. Consequently ring-shaped unstable sets play a central role for dynamical transitions occurring near the tongues. Finally, we note that these kinds of dynamical transitions seem to be "universal," in that we observe that they occur in typical quasiperiodically forced period-doubling systems of different nature, such as the quasiperiodically forced Hénon map, ring map, and pendulum [21].

S.Y.K. thanks A. Jalnine for fruitful discussions on dynamical transitions in quasiperiodically forced systems. This work was supported by the Korea Research Foundation (Grant No. KRF-2001-013-D00014) and the National Science Foundation.

* Electronic address: sykim@kangwon.ac.kn

$\dagger$ Department of Physics and Department of Electrical and Computing Engineering, University of Maryland, College
Park, Maryland 20742

[1] A. Prasad, S. S. Negi and R. Ramaswamy, Int. J. Bif. Chaos 11, 291 (2001).

[2] C. Grebogi, E. Ott, S. Pelikan, and J. A. Yorke, Physica D 13, 261 (1984).

[3] F. J. Romeiras and E. Ott, Phys. Rev. A 35, 4404 (1987); M. Ding, C. Grebogi, and E. Ott, Phys. Rev. A 39, 2593 (1989).

[4] T. Kapitaniak, E. Ponce, and T. Wojewoda, J. Phys. A 23, L383 (1990).

[5] J. F. Heagy and S. M. Hammel, Physica D 70, 140 (1994)

[6] A. S. Pikovsky and U. Feudel, Chaos 5, 253 (1995). See Eqs. (11)-(14) for the definition of $\delta$.

[7] U. Feudel, J. Kurths, A. S. Pikovsky, Physica D 88, 176 (1995); O. Sosnovtseva, U. Feudel, J. Kurths, and A. Pikovsky, Phys. Lett. A 218, 255 (1996); U. Feudel, C. Grebogi, and E. Ott, Phys. Rep. 290, 11 (1997).

[8] S. Kuznetsov, A. Pikovsky, and U. Feudel, Phys. Rev. E 51, R1629 (1995); S. Kuznetsov, U. Feudel, and A. Pikovsky, Phys. Rev. E 57, 1585 (1998); S. Kuznetsov, E. Neumann, A. Pikovsky, and I. Sataev, Phys. Rev. E 62, 1995 (2000).

[9] P. R. Chastell, P. A. Glendinning, and J. Stark, Phys. Lett. A 200, 17 (1995); H. Osinga, J. Wiersig, P. Glendinning, and U. Feudel, Int. J. Bif. Chaos 11, 3085 (2001); B. R. Hunt and E. Ott, Phys. Rev. Lett. 87, 254101 (2001).

[10] T. Nishikawa and K. Kaneko, Phys. Rev. E 54, 6114 (1996)

[11] T. Yalçınkaya and Y.-C. Lai, Phys. Rev. Lett. 77, 5039 (1996).

[12] A. Prasad, V. Mehra, and R. Ramaswamy, Phys. Rev. Lett. 79, 4127 (1997); Phys. Rev. E 57, 1576 (1998).

[13] A. Witt, U. Feudel, and A. Pikovsky, Physica D 109, 180 (1997).

[14] A. Venkatesan, M. Lakshmanan, A. Prasad and R. Ramaswamy, Phys. Rev. E 61, 3641 (2000); A. Venkatesan and M. Lakshmanan, Phys. Rev. E 63, 026219 (2001).

[15] S. S. Negi, A. Prasad, and R. Ramaswamy, Physica D 145, 1 (2000).

[16] H. M. Osinga and U. Feudel, Physica D 141, 54 (2000).

[17] W. L. Ditto, M. L. Spano, H. T. Savage, S. N. Rauseo, J. Heagy, and E. Ott, Phys. Rev. Lett. 65, 533 (1990); T. Zhou, F. Moss, and A. Bulsara, Phys. Rev. A 45, 5394 (1992); W. X. Ding, H. Deutsch, A. Dinklage, and C. Wilke, Phys. Rev. E 55, 3769 (1997); T. Yang and K. Bilimgut, Phys. Lett. A 236, 494 (1997); B. P. Bezruchko, S. P. Kuznetosv, and Y. P. Seleznev, Phys. Rev. E 62, 7828 (2000).

[18] A. Bondeson, E. Ott, and T. M. Antonsen, Phys. Rev. Lett. 55, 2103 (1985); J. A. Ketoja and I. I. Satija, Physica D 109, 70 (1997).

[19] C.-S. Zhou and T.-L. Chen, Europhys. Lett. 38, 261 (1997); R. Ramaswamy, Phys. Rev. E 56, 7294 (1997).

[20] C. Mira, L. Gardini, A. Barugola, and J.-C. Cathala, Chaotic Dynamics in Two-Dimensional Noninvertible Maps (World Scientific, Singapore, 1996).

[21] S.-Y. Kim, W. Lim, and E. Ott (unpublished).

[22] K. Kaneko, Prog. Theor. Phys. 72, 202 (1984). 